Methods The Medical Outcomes Study Short Form (SF-36) and the Lupus Quality of life (LupusQol) were applied in a cohort of 38 SLE patients. At the time of HRQOL testing, all patients underwent a clinical and laboratory evaluation, together with the measure of disease activity using the Systemic Lupus Erythematous Disease Activity Index (SLEDAI$2 \mathrm{~K})$. In addition, a battery of psychological tests including the Hamilton Anxiety Scale (HAS) and the Hamilton Depression Rating Scale (HAM-D) was applied.

Results The parameters which seemed to greatly influence the impairment of HRQOL were female gender, marital statues, a higher SLEDAI-2k scores as well as higher HAS and HAM-D scores. Arthralgia-arthritis, cutaneous disease activity, neurological disease activity and renal disease activity were correlated negatively with LupusQol subscales. There was a strong positive correlation beteween comparable domains of instruments. Although not as strong as comparable domains, significant correlations were also found between noncomparable domains of LupusQol and PCS and MCS of SF-36.

Conclusions SF-36 and LupusQol were both beneficial instruments in evaluating HRQOL of Tunisian patients with SLE. Anxiety, depression and disease activity in some organs seem to be the major deteminants of HRQOL impairment in SLE patients.

Funding Source(s): None

\section{BLOOD CONCENTRATIONS OF COMPLEMENT SPLIT PRODUCT IC3B AND SERUM C3 ASSOCIATE WITH SYSTEMIC LUPUS ERYTHEMATOSUS DISEASE ACTIVITY}

${ }^{1}$ Alfred H Kim*, ${ }^{2}$ Vibeke Strand, ${ }^{1}$ Deepali P Sen, ${ }^{3}$ Qiang J Fu, ${ }^{4}$ Martin J Schmidt, ${ }^{1}$ John P Atkinson. 'Washington University School of Medicine; ${ }^{2}$ Stanford University School of Medicine; ${ }^{3}$ Saint Louis University College for Public Health and Social Justice; ${ }^{4}$ Kypha, Inc

\subsection{6/lupus-2019-Ism.54}

Background A major unmet need in SLE is the identification of a biomarker that consistently tracks with disease activity. One current approach is measuring complement activation by evaluating consumption of serum C3 and C4. However, since they are acute phase reactants, interpretation of these levels is challenging as serum levels may not decrease until late in a disease flare. $\mathrm{iC} 3 \mathrm{~b}$ is a proteolytically derived molecule of $\mathrm{C} 3 \mathrm{~b}$ and increases with complement activation. $\mathrm{iC} 3 \mathrm{~b} / \mathrm{C} 3$ ratio measures complement consumption relative to production, which may provide for a more accurate assessment of complement activation. We hypothesize that blood iC3b and iC $3 b / C 3$ levels will provide a more specific and reliable marker of complement activation and disease activity in SLE.

Methods 159 consecutive subjects with American College of Rheumatology or Systemic Lupus International Collaborating Clinics classified SLE were enrolled into CASTLE (Complement Activation Signatures in Systemic Lupus Erythematosus), a prospective observational study. Patients with 1-7 study visits were included in this longitudinal analysis. 48 healthy volunteers were enrolled to establish the normal reference iC3b/ C3 ratio. Serum C3 and C4 were measured by nephelometry and blood iC3b levels by a lateral flow assay. SLE disease activity was monitored utilizing the Systemic Lupus Erythematosus Disease Activity Index 2K Responder Index-50 instrument.

Results iC3b/C3 ratio, double-stranded (ds)DNA antibodies (Abs), and supraphysiologic prednisone dose (>7.5 mg/day) each independently correlated with SLE disease activity, employing multilevel multiple logistic regression analysis. Only the $\mathrm{iC} 3 \mathrm{~b} / \mathrm{C} 3$ ratio was significantly associated with clinically meaningful improvements in disease activity among subjects receiving supraphysiologic doses of prednisone. iC3b/C3 outperformed C3 and C4 levels discriminating both active versus inactive SLE disease and major flares versus no disease activity. $\mathrm{iC} 3 / \mathrm{C} 3$, dsDNA Abs, ESR, and supraphysiologic prednisone dose were independently associated with lupus nephritis, while none were associated with SLE rash. The association of $\mathrm{iC} 3 \mathrm{~b} /$ C3 with nephritis was independent of other observed clinical manifestations. Finally, we observed a stronger association of the $\mathrm{iC} 3 \mathrm{~b} / \mathrm{C} 3$ ratio with SLE disease activity in African-Americans compared to Whites.

Conclusions Blood $\mathrm{iC} 3 \mathrm{~b} / \mathrm{C} 3$ correlates with SLE disease activity and clinically meaningful changes. Furthermore, it discriminates between active versus inactive SLE, and major flares compared to those patients without active disease. Differences in the strength of association was observed between races and manifestations.

Funding Source(s): Kypha, Inc. and National Institutes of Health (NIH)/National Institute of Arthritis and Musculoskeletal and Skin Diseases (NIAMS) under Award Number R21AR069833.

\section{POOR SLEEP QUALITY ASSESSED SUBJECTIVELY ASSOCIATED WITH WORSENING SLE DISEASE ACTIVITY}

${ }^{1}$ Alicia M Hinze, ${ }^{2}$ Philip Chu, ${ }^{3}$ Deepali P Sen, ${ }^{3}$ Noor Al-Hammadi, ${ }^{3}$ Yo-El S Ju, ${ }^{3}$ Alfred H Kim*. 'Mayo Clinic College of Medicine; '2Duke University School of Medicine; ${ }^{3}$ Washington University School of Medicine

\subsection{6/lupus-2019-Ism.55}

Background Poor sleep quality is commonly observed in patients with SLE. We hypothesize that poor sleep contributes to worsening SLE. The aims of this study are to evaluate the relationship between subjective sleep measures and SLE activity over time.

Methods A prospective, observational study evaluated the relationship between sleep and SLE disease activity. 151 patients were enrolled. Pittsburgh

Sleep Quality Index (PSQI), Epworth Sleepiness Scale (ESS), Patient Reported Outcomes Measurement Instrument System (PROMIS)-Sleep Related Impairment (SRI), and PROMIS-Sleep Disturbance (SD) survey instruments measured patient reported sleep quality. The population mean for the PROMIS instruments is 50. The SLEDAI-2000 Responder Index-50 (S2K RI-50) was used to define active SLE as S2K RI-50 >4 and worsening SLE at subsequent visits as an increase in S2K RI-50 $\geq 4$. Baseline comparisons were calculated using non-parametric tests. Kaplan-Meier examined the relationship between poor sleep and worsening SLE activity over time.

Results At baseline, the median age was 42, 90.7\% were female, 54.3\% were African American, 24.5\% were on prednisone doses $>7.5 \mathrm{mg} / \mathrm{day}$, and $36.4 \%$ had active SLE. Patients with active SLE had significantly higher SRI scores (median 64.3) vs inactive SLE (median 56.6) as well as significantly higher SD scores (median 58.3 vs 52.2), whereas PSQI and ESS were not significantly different.

Data from 109 patients with $\geq 2$ visits were used for longitudinal studies. Kaplan-Meier analysis, stratified by SRI T-score of $>60$ vs $\leq 60$ demonstrated that worse sleep $($ SRI $>60)$ at the 


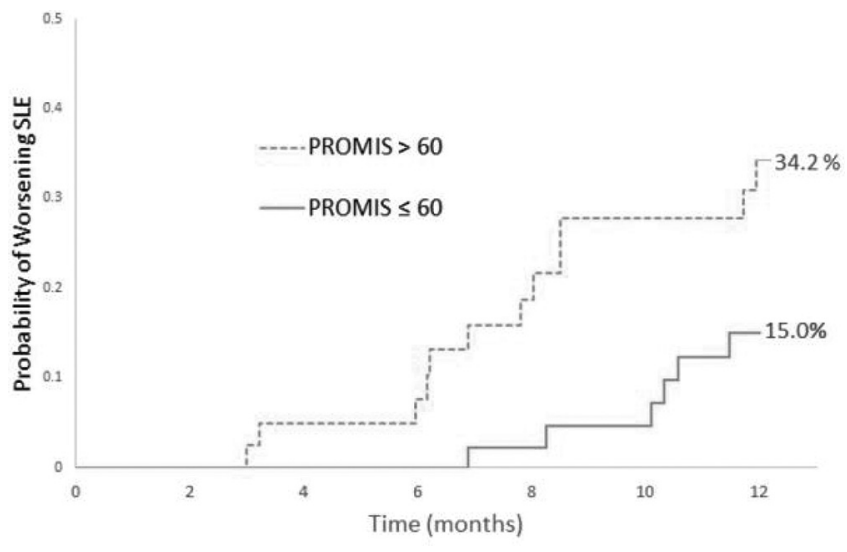

Abstract 55 Figure 1 Poor sleep quality at prior visit predicts SLE flare activity

previous visit predicted worsening SLE activity at the next visit (figure 1). Over a 12 month period, the probability of SLE activity worsening was $21.4 \%$ overall, $34.2 \%$ for SRI $>60$, and $15.0 \%$ for SRI $\leq 60(p=0.024)$.

Conclusions Our study reinforces that patients with SLE report worse subjective sleep compared to the general population, and patients with active SLE have worse sleep than patients with inactive SLE. Our longitudinal data demonstrate that poor sleep predicts worsening SLE disease activity. Thus, variation in subjective sleep may have an important role in SLE flares.

Funding Source(s): AMH and PC: Washington University School of Medicine Mentors in Medicine Program, Rheumatology Research Foundation Resident Research Preceptorship Award. YSJ: NIH/NINDS K23NS089922, NIH/NIA R34AG056639, UL1RR024992, and KL2-TR000450. AHJK: NIH/NIAMS R21AR069833, Midwest Strategic Pharma-Academic Research Consortium, and the Doris Duke Foundation Fund for Retaining Clinical Scientists Program.

\section{SELF-REPORTED INDIRECT COSTS ARE UNDERESTIMATED IN A CANADIAN COHORT OF PATIENTS WITH SLE}

${ }^{1}$ Ann E Clarke*, ${ }^{1}$ Megan RW Barber, ${ }^{2}$ Yvan St Pierre, ${ }^{3}$ Murray B Urowitz, ${ }^{3}$ Dafna D Gladman, ${ }^{2}$ Sasha Bernatsky, ${ }^{2}$ Evelyne Vinet, ${ }^{2}$ Christian Pineau, ${ }^{4} J o h n$ Hanly, ${ }^{5}$ Christine Peschken, ${ }^{6}$ Paul Fortin, ${ }^{1}$ Michelle Jung, ${ }^{1}$ Claire Barber, ${ }^{7}$ Susan Elliott. ${ }^{1}$ Division of Rheumatology, Cumming School of Medicine, University of Calgary; ${ }^{2}$ Department of Medicine, Division of Rheumatology, Faculty of Medicine, McGill University; ${ }^{3}$ Krembil Research Institute, University of Toronto; ${ }^{4}$ Division of Rheumatology, Department of Medicine, Dalhousie University; ${ }^{5}$ Faculty of Medicine, Department of Internal Medicine, University of Manitoba; ${ }^{6}$ Centre de Recherche, Université Laval; ${ }^{7}$ Department of Geography and Environmental Management, University of Waterloo

\subsection{6/lupus-2019-Ism.56}

Background Indirect costs (IDC) of SLE reflect lost productivity in work force (WF) and non-WF activities and can be expressed as: 1) patient self-report of lost productivity or 2) the difference between productivity of an age-and-sex matched general population and the patients stated productivity. We assess IDC calculated by both methods in a Canadian-wide cohort and compare IDC, stratified by damage, across methods. Methods Patients fulfilling the ACR or SLICC Classification Criteria from 6 centres were enrolled. Participants completed a validated questionnaire on lost productivity. Lost
Abstract 56 Table 1 Indirect cost calculations by Method \#1 (difference between patient self-report of expected versus actual productivity) and Method \#2 (difference between time worked by matched general population versus actual patient productivity)

\begin{tabular}{llll}
\hline SDI & $\begin{array}{l}\text { IDC Method \#1 } \\
\text { Mean }(95 \% \mathrm{Cl})\end{array}$ & $\begin{array}{l}\text { IDC Method \#2 } \\
\text { Mean }(95 \% \mathrm{Cl})\end{array}$ & $\begin{array}{l}\text { Difference between } \\
\text { Method \#1 and \#2 } \\
\text { Mean }(95 \% \mathrm{Cl})\end{array}$ \\
\hline $\mathbf{0}$ & $\$ 17109$ & $\$ 19326$ & $\$ 2217^{1}$ \\
1 & $(\$ 13,021, \$ 21,197)$ & $(\$ 14,936, \$ 23,717)$ & $(\$-3782, \$ 8216)$ \\
& $\$ 19937$ & $\$ 25963$ & $\$ 6027^{2}$ \\
2 & $(\$ 16,158, \$ 23,715)$ & $(\$ 21,764, \$ 30,163)$ & $(\$ 378, \$ 11,676)$ \\
& $\$ 22825$ & $\$ 31733$ & $\$ 8908$ \\
3 & $(\$ 18,479, \$ 27,171)$ & $(\$ 27,401, \$ 36,064)$ & $(\$ 2772, \$ 15,044)$ \\
4 & $(\$ 19398$ & $\$ 30022$ & $\$ 10624$ \\
& $\$ 26159$ & $(\$ 23,682, \$ 36,362)$ & $(\$ 2293, \$ 18,956)$ \\
$\geq 5$ & $(\$ 19,195, \$ 33,124)$ & $(\$ 29,819, \$ 45,605)$ & $(\$ 1027, \$ 22,079)$ \\
& $\$ 25265$ & $\$ 41220$ & $\$ 15,955,2$ \\
& $(\$ 19,647, \$ 30,883)$ & $(\$ 35,456, \$ 46,984)$ & $(\$ 7906, \$ 24,004)$ \\
\hline
\end{tabular}

${ }^{1}$ The difference between the between method difference at $\mathrm{SDI} \geq 5$ and $\mathrm{SDI}=0$ is $\$ 13738$ $(95 \% \mathrm{Cl}, \$ 3700, \$ 23,777)$.

${ }^{2}$ The difference between the between method difference at $\mathrm{SDI} \geq 5$ and $\mathrm{SDI}=1$ is $\$ 9928$ $(95 \% \mathrm{Cl}, \$ 95, \$ 19,762)$.

productivity was calculated as: 1) the difference between the time patients reported they expected they would engage in WF and non-WF activities if not ill versus the time they reported working and 2) the difference between the time worked by an age-and-sex matched general population in WF and non-WF activities versus the time patients reported working. IDC were valued using age-and-sex-specific wages from the Statistics Canada General Social Survey. IDC from nonWF activities were valued using opportunity costs (i.e., expected WF earnings, rather than expected earning of service workers). Annual IDC (2017 Canadian dollars) associated with damage measured on the SLICC/ACR Damage Index (SDI) were obtained from multiple regressions adjusting for age, race/ethnicity, and disease duration.

Results 1368 patients participated, 90.4\% female, 70.9\% Caucasian, mean age at diagnosis 33.0 years (SD 13.5), mean SLE duration 16.8 years (SD 11.6), mean SLE Disease Activity Index (SLEDAI-2K) 2.15 (SD 3.07), and mean SDI 1.54 (SD 1.87). IDC by method \#1 versus \#2, stratified by SDI, are shown in table 1 . Although at $\mathrm{SDI}=0$, mean predicted IDC did not differ between methods, for $\mathrm{SDI}=1$ through SDI 5, IDC by method \#2 were greater.

Conclusions IDC by method \#2 were greater for SDIs 1 through 5 and the difference between methods increased significantly between lower and higher SDIs ( $<2$ versus 5). Our results suggest that IDC calculated by comparing the patients actual productivity to their self-report of expected productivity versus the productivity of an age-and-sex-matched general population leads to underestimation, which is not associated with damage. Patients expectations of productivity appear to plateau with increasing damage and do not reflect their likely productivity if they were not ill. Hence, IDC should not only rely on patients self-report of lost productivity, but should also incorporate a comparison of the patients productivity with the actual productivity of a matched general population. Funding Source(s): Canadian Initiative for Outcomes in Rheumatology cAre (CIORA) 